\title{
Reversible Pulmonary Hypertension Related to Thalidomide Treatment for Multiple Myeloma
}

\author{
Antonio Villa ${ }^{a}$ Anna Alice Mazzola ${ }^{b}$ Stefano Ghioc \\ Elena Martinoli ${ }^{\mathrm{a}}$ Pietro Marino \\ aU.O. Medicina d'Urgenza e bU.O. Cardiologia, Ospedale Fatebenefratelli, Milano, e \\ 'Dipartimento di Cardiologia, IRCCS Policlinico San Matteo, Pavia, Italia
}

\section{Key Words}

Pulmonary hypertension $\cdot$ Multiple myeloma $\cdot$ Thalidomide

\begin{abstract}
Multiple myeloma (MM) is thrombogenic as a consequence of multiple hemostatic effects. Thalidomide is an effective treatment; however, it has been associated with an increased risk of thromboembolic events including pulmonary hypertension (PH). $\mathrm{PH}$ in the absence of thromboembolic events has also been described in some patients with $\mathrm{MM}$ during thalidomide treatment. We experienced occurrence of $\mathrm{PH}$ in a MM patient during treatment with thalidomide. A 79-year-old woman was diagnosed with IgG lambda MM and was started on thalidomide treatment. About a month later, she presented with asthenia, palpitation and dyspnoea on exertion and was hospitalized. An echocardiography revealed severe $\mathrm{PH}$ (systolic pulmonary artery pressure $75 \mathrm{~mm} \mathrm{Hg}$ ) without paradoxic movement of ventricular septum or right ventricular dysfunction signs; a previous echocardiography was normal. Pulmonary computed tomography and perfusion scan were negative for pulmonary embolism. Based on the hypothesis of a pharmacological pathogenesis, thalidomide was promptly interrupted. About a month later, she was hospitalized for further investigations. Physical examination documented absence of dyspnoea or other respiratory signs, and echocardiography showed normal right ventricular function and normal pulmonary artery pressure. As in the cases reported in the literature, we suggest a possible direct correlation between thalidomide and $\mathrm{PH}$, since in all cases a rapid decrease of pulmonary artery pressure after thalidomide discontinuation was observed.
\end{abstract}




\section{Introduction}

Multiple myeloma (MM) is thrombogenic as a consequence of multiple hemostatic effects, including elevated interleukin-6 levels, procoagulant antibody formation, paraprotein interference with fibrin structure, activated protein $\mathrm{C}$ resistance and endothelial damage [1].

Thalidomide is an effective treatment both in advanced MM and as first-line therapy in combination with dexamethasone or other cytotoxic chemotherapy [2]; it has been associated with an increased risk of thromboembolic events including pulmonary hypertension $(\mathrm{PH})[3,4]$. PH in the absence of thromboembolic events has also been described in 3 patients with MM during thalidomide treatment [5-7] and in a pilot study [1]. We also experienced occurrence of $\mathrm{PH}$ in a MM patient during treatment with thalidomide.

\section{Case Presentation}

In September 2010, a 79-year-old woman was diagnosed with IgG lambda MM evolved from monoclonal gammopathy of unknown significance (MGUS). In April 2011, the patient was started on thalidomide (50 mg daily) and prednisone treatment. About a month later, she presented with asthenia, palpitation and dyspnoea on exertion and was hospitalized.

Physical examination revealed mild tachypnea (20 breaths/min) and tachyarrhythmia. Cyanosis and edema of the lower extremities were not found. ECG showed tachyarrhythmia with atrial fibrillation (140 beats/min). Within $24 \mathrm{~h}$, a sinus rhythm was restored with atenolol. A blood sample analysis yielded the following results: NT-pro-BNP 3,021 ng/l (normal value <450), D-dimer $519 \mathrm{ng} / \mathrm{ml}$ (normal value $<300$ ), and T-troponin $37.2 \mathrm{ng} / \mathrm{l}$ (normal value $<14$ ). Blood arterial gas analysis parameters were: $\mathrm{pH} 7.49, \mathrm{pO}_{2} 70.2 \mathrm{~mm} \mathrm{Hg}, \mathrm{pCO}_{2} 25.1 \mathrm{~mm} \mathrm{Hg}, \mathrm{HCO}_{3}{ }^{-} 19.2 \mathrm{mmol} / \mathrm{l}, \mathrm{SatO}_{2}$ 95.7\%, $\mathrm{pO}_{2} / \mathrm{FiO}_{2} 336 \mathrm{~mm} \mathrm{Hg}$.

An echocardiography performed a few days later showed severe $\mathrm{PH}$ (systolic pulmonary artery pressure $75 \mathrm{~mm} \mathrm{Hg}$ ) with mild tricuspid insufficiency without paradoxic movement of ventricular septum or right ventricular dysfunction signs, and normal left ventricular function. A previous echocardiography done in 2008 was normal. Pulmonary computed tomography and perfusion scan were negative for pulmonary embolism. Functional lung investigations showed severe oxygen desaturation in a 6-min-walk test. No COPD history was present. Connective tissue disease was also excluded by further examination. Based on the hypothesis of a pharmacological pathogenesis, thalidomide was promptly interrupted. The patient was sent to the Center for Pulmonary Hypertension (Policlinico San Matteo, Pavia, Italy) for further investigations.

In June 2011 (about a month later), she was hospitalized. Physical examination revealed absence of dyspnoea or other respiratory signs. Sinus rhythm on ECG and echocardiography showed normal right ventricular function and normal pulmonary artery pressure. A clinical control in July 2011 confirmed absence of symptoms, and showed a peripheral $\mathrm{SatO}_{2}$ of $97 \%$ and a pulmonary artery pressure of $26 \mathrm{~mm}$ $\mathrm{Hg}$.

\section{Discussion}

As in the 3 cases reported in the literature [5-7], we suggest a possible direct correlation between thalidomide and $\mathrm{PH}$, since in all cases a rapid decrease of pulmonary artery pressure after thalidomide discontinuation was observed. Furthermore, in the pilot study of Lafaras et al. [1], 4 of the 82 treated patients had PH and improved after discontinuation of therapy. Our patient showed clinical and echocardiographic signs of $\mathrm{PH}$ after a few days of thalidomide therapy. In the described case reports, symptoms 
occurred after a few months, but in the cohort described by Lafaras et al. [1], 2 patients showed subclinical PH 1 month after thalidomide administration.

The etiology of $\mathrm{PH}$ in these patients is still unclear. Adverse effects of thalidomide should be considered in the absence of pulmonary embolism. Thalidomide possibly causes a vasodilator and vasoconstriction imbalance, which may cause abnormal pulmonary vascular response leading to a vicious circle perpetuating $\mathrm{PH}[8,9]$. A direct effect of thalidomide on the pulmonary endothelial cells could be postulated [5]. In the case described by Hattori et al. [6], autopsy revealed an extensive formation of plexogenic pulmonary arteries accompanied by thickening of intimae and media arteries. Lafaras et al. [1] indicate that the subgroup of MM patients with unknown heart disease (coronary artery disease, valvular heart disease and hypertension) receiving thalidomide should be thoroughly examined by echocardiography for the early detection of clinical and/or subclinical $\mathrm{PH}$.

Thus, echocardiographic evaluation might be necessary to all patients receiving thalidomide; it not only provides an estimate of pulmonary pressure but also may help to exclude any secondary causes of $\mathrm{PH}$, predict the prognosis, monitor the efficacy of specific therapeutic interventions and detect the preclinical stage of the disease [1].

\section{References}

1 Lafaras C, Mandala E, Verrou E, Platogiannis D, Barbetakis N, Bischiniotis T, Zervas K: Non-thromboembolic pulmonary hypertension in multiple myeloma, after thalidomide treatment: a pilot study. Ann Oncol 2008;19:1765-1769.

-2 Glasmacher A, Hahn C, Hoffmann F, Naumann R, Goldschmidt H, von Lilienfeld-Toal M, Orlopp K, SchmidtWolf I, Gorschlüter M: A systematic review of phase-II trials of thalidomide monotherapy in patients with relapsed or refractory multiple myeloma. Br J Haematol 2006;132:584-593.

-3 Cavo M, Zamagni E, Cellini C, Tosi P, Cangini D, Cini M, Valdrè L, Palareti G, Masini L, Tura S, Baccarani M: Deep vein thrombosis in patients with multiple myeloma receiving first line thalidomide-dexamethasone therapy. Blood 2002;100:2272-2273.

-4 Osman K, Comenzo R, Rajkumar SV: Deep vein thrombosis and thalidomide therapy for multiple myeloma. N Engl J Med 2001;344:1951-1952.

5 Younis TH: Reversible pulmonary hypertension and thalidomide therapy for multiple myeloma. Br J Haematol 2003;121:191-192.

6 Hattori Y, Shimoda M, Okamoto S, Satoh T, Kakimoto T, Ikeda Y: Pulmonary hypertension and thalidomide therapy in multiple myeloma. Br J Haematol 2005;128:885-888.

7 Antonioli E, Nozzoli C, Gianfaldoni G, Mannelli F, Rossi S, Betti S, Bernardeschi P, Fiorentini G, Bosi A: Pulmonary hypertension related to thalidomide therapy in refractory multiple myeloma. Ann Oncol 2005;16:1849-1850.

8 Pepke-Zaba J, Morrell NW: The endothelin system and its role in pulmonary arterial hypertension (PAH) Thorax 2005;60:443-444.

9 Steiner MK, Preston IR, Klinger Jr, Hill NS: Pulmonary hypertension: inhaled nitric oxide, sildenafil and natriuretic peptides: Curr Opin Pharmacol 2005;5:245-250. 